\title{
Weak solutions for a system of quasilinear elliptic equations
}

\author{
Maria Alessandra Ragusa ${ }^{1,2, *}$, Abdolrahman Razani $^{3}$ \\ ${ }^{1}$ Dipartimento di Matematica e Informatica, Università di Catania, Catania 95125, Italy \\ ${ }^{2}$ Faculty of Science, RUDN University, Moscow 117198, Russia \\ ${ }^{3}$ Department of Pure Mathematics, Faculty of Science, Imam Khomeini International University, Qazvin 34149-16818, Iran
}

(Received: 5 June 2020. Accepted: 1 August 2020. Published online: 4 August 2020.)

(C) 2020 the authors. This is an open access article under the CC BY (International 4.0) license (www.creativecommons.org/licenses/by/4.0/).

\begin{abstract}
A system of quasilinear elliptic equations on an unbounded domain is considered. The existence of a sequence of radially symmetric weak solutions is proved via variational methods.
\end{abstract}

Keywords: sequence of solutions, elliptic problem, $p$-Laplacian, variational methods.

2020 Mathematics Subject Classification: 34B10, 35J20, 35J50.

\section{Introduction}

We consider the following problem

$$
\begin{cases}-\Delta_{p} u+|u|^{p-2} u=\lambda \alpha_{1}(x) f_{1}(v) & \text { in } \mathbb{R}^{N}, \\ -\Delta_{q} v+|v|^{q-2} v=\lambda \alpha_{2}(x) f_{2}(u) & \text { in } \mathbb{R}^{N}, \\ u, v \in W^{1, p}\left(\mathbb{R}^{N}\right), & \end{cases}
$$

where $p, q>N>1$. We assume that $f_{1}, f_{2}: \mathbb{R} \rightarrow \mathbb{R}$ are continuous functions, $\alpha_{1}(x), \alpha_{2}(x) \in L^{1}\left(\mathbb{R}^{N}\right) \cap L^{\infty}\left(\mathbb{R}^{N}\right)$ are nonnegative (not identically zero) radially symmetric maps, and $\lambda$ is a real parameter. Also $\Delta_{p} u:=\operatorname{div}\left(|\nabla u|^{p-2} \nabla u\right)$ denotes the $p$-Laplacian operator.

Partial differential equations are used to model a wide variety of physically significant problems arising in different areas such as physics, engineering and other applied disciplines (see [7, 11, 12, 18, 25, 26, 28-31, 34-45]). Sobolev spaces play an important role in the theory of partial differential equations as well as Orlicz-Morrey space and $\dot{B}_{\infty, \infty}^{-1}$ space (see [2, 8-10,32-34]). Laplace equation is the prototype for linear elliptic equations. This equation has a non-linear counterpart, the so-called $p$-Laplace equation (see [1,6,13,14,19,21-24,48]).

Here, by inspiration of [20], we prove the existence of a sequence of radially symmetric weak solutions for (1) in the unbounded domain $\mathbb{R}^{N}$.

The solution of (1) belongs to the product space

$$
W^{1,(p, q)}\left(\mathbb{R}^{N}\right)=W^{1, p}\left(\mathbb{R}^{N}\right) \times W^{1, q}\left(\mathbb{R}^{N}\right)
$$

equipped with the norm $\|(u, v)\|_{(p, q)}=\|u\|_{p}+\|u\|_{q}$.

Definition 1.1. For fixed $\lambda,(u, v): \mathbb{R}^{\mathbb{N}} \rightarrow \mathbb{R}$ is said to be a weak solution of $(1)$, if $(u, v) \in W^{1,(p, q)}\left(\mathbb{R}^{N}\right)$ and for every $(z, w) \in W^{1,(p, q)}\left(\mathbb{R}^{N}\right)$

$$
\begin{gathered}
-\int_{\mathbb{R}^{N}}|\nabla u(x)|^{p-2} \nabla u(x) \cdot \nabla z(x) d x-\int_{\mathbb{R}^{N}}|\nabla u(x)|^{q-2} \nabla u(x) \cdot \nabla w(x) d x+\int_{\mathbb{R}^{N}}|u(x)|^{p-2} u(x) z(x) d x+\int_{\mathbb{R}^{\mathbb{N}}}|v(x)|^{q-2} v(x) w(x) d x \\
-\lambda \int_{\mathbb{R}^{N}} \alpha_{1}(x) f_{1}(v(x)) z(x) d x-\lambda \int_{\mathbb{R}^{N}} \alpha_{2}(x) f_{2}(u(x)) w(x) d x=0,
\end{gathered}
$$

where

$$
\|(u, v)\|_{W^{1,(p, q)}\left(\mathbb{R}^{N}\right)}:=\left(\int_{\mathbb{R}^{\mathbb{N}}}|\nabla u(x)|^{p} d x+\int_{\mathbb{R}^{\mathbb{N}}}|u(x)|^{p} d x\right)+\left(\int_{\mathbb{R}^{\mathbb{N}}}|\nabla v(x)|^{q} d x+\int_{\mathbb{R}^{\mathbb{N}}}|v(x)|^{q} d x\right) .
$$

\footnotetext{
${ }^{*}$ Corresponding author (maragusa@dmi.unict.it)
} 
Note that the critical points of a energy functional are exactly the weak solutions of (1).

Morrey's theorem, implies the continuous embedding

$$
W^{1,(p, q)}\left(\mathbb{R}^{\mathbb{N}}\right) \hookrightarrow L^{\infty}\left(\mathbb{R}^{\mathbb{N}}\right) \times L^{\infty}\left(\mathbb{R}^{\mathbb{N}}\right),
$$

which says that there exists $c$ (depends on $p, q, N)$, such that $\|(u, v)\|_{\infty} \leq c\|(u, v)\|_{W^{1,(p, q)}\left(\mathbb{R}^{N}\right)}$, for every $(u, v) \in W^{1, p}\left(\mathbb{R}^{\mathbb{N}}\right) \times$ $W^{1, q}\left(\mathbb{R}^{\mathbb{N}}\right)$, where $\|(u, v)\|_{\infty}:=\max \left\{\|u\|_{\infty},\|v\|_{\infty}\right\}$. Since in the low-dimensional case, every function $(u, v) \in W^{1,(p, q)}\left(\mathbb{R}^{N}\right)$ admits a continuous representation (see [4, p.166]). In the sequel we will replace $(u, v)$ by this element.

We need the following notations (see [5] or [17] for more details):

(I) $O(N)$ stands for the orthogonal group of $\mathbb{R}^{N}$.

(II) $B(0, s)$ denotes the open $N$-dimensional ball of center zero, radius $s>0$ and standard Lebesgue measure, meas $(B(0, s))$.

(III) $\|\alpha\|_{B\left(0, \frac{s}{2}\right)}:=\int_{B\left(0, \frac{s}{2}\right)} \alpha(x) d x$.

\section{Definition 1.2.}

- A function $h: \mathbb{R}^{N} \rightarrow \mathbb{R}$ is radially symmetric if $h(g x)=h(x)$, for every $g \in O(N)$ and $x \in \mathbb{R}^{N}$.

- Let $G$ be a topological group. A continuous map $\xi: G \times X \rightarrow X:(g, x) \rightarrow \xi(g, u):=g u$, is called the action of $G$ on the Banach space $\left(X,\|\cdot\|_{X}\right)$ if

$$
1 u=u, \quad(g m) u=g(m u), \quad u \mapsto g u \text { is linear. }
$$

- The action is said to be isometric if $\|g u\|_{X}=\|u\|_{X}$, for every $g \in G$.

- The space of G-invariant points is defined by

$$
F i x(G):=\{u \in X: g u=u, \text { for all } g \in G\} \text {. }
$$

- A map $m: X \rightarrow \mathbb{R}$ is said to be $G$-invariant if $m o g=m$ for every $g \in G$.

The following theorem is important to study the critical point of the functional.

Theorem 1.1. [27] Assume that the action of the topological group $G$ on the Banach space $X$ is isometric. If $J \in C^{1}(X: \mathbb{R})$ is G-invariant and if $u$ is a critical point of $J$ restricted to Fix $(G)$, then u is a critical point of $J$.

The action of the group $O(N)$ on $W^{1, p}\left(\mathbb{R}^{N}\right)$ can be defined by $(g u)(x):=u\left(g^{-1} x\right)$, for every $g \in W^{1, p}\left(\mathbb{R}^{N}\right)$ and $x \in \mathbb{R}^{\mathbb{N}}$. A computation shows that this group acts linearly and isometrically, which means $\|u\|=\|g u\|$, for every $g \in O(N)$ and $u \in W^{1, p}\left(\mathbb{R}^{N}\right)$.

Definition 1.3. The subspace of radially symmetric functions of $W_{r}^{1,(p, q)}\left(\mathbb{R}^{N}\right)$ is defined by

$$
\begin{aligned}
X & :=W_{r}^{1,(p, q)}\left(\mathbb{R}^{N}\right) \\
& :=\left\{(u, v) \in W^{1,(p, q)}\left(\mathbb{R}^{N}\right):\left(g_{1} u, g_{2} v\right)=(u, v), \text { for all }\left(g_{1}, g_{2}\right) \in O(N) \times O(N)\right\},
\end{aligned}
$$

and endowed by the norm

$$
\|(u, v)\|_{W_{r}^{1,(p, q)}\left(\mathbb{R}^{N}\right)}:=\left(\int_{\mathbb{R}^{\mathbb{N}}}|\nabla u(x)|^{p} d x+\int_{\mathbb{R}^{\mathbb{N}}}|u(x)|^{p} d x\right)+\left(\int_{\mathbb{R}^{\mathbb{N}}}|\nabla v(x)|^{q} d x+\int_{\mathbb{R}^{\mathbb{N}}}|v(x)|^{q} d x\right) .
$$

In what follows: $\|(u, v)\|_{r}$ denotes $\|(u, v)\|_{W_{r}^{1,(p, q)}\left(\mathbb{R}^{N}\right)}$.

The following crucial embedding result due to Kristály and principally based on a Strauss-type estimation (see [46]) (Also see [15, Theorem 3.1], and [16,47] for related subjects).

Theorem 1.2. The embedding $W_{r}^{1, p}\left(\mathbb{R}^{N}\right) \hookrightarrow L^{\infty}\left(\mathbb{R}^{N}\right)$, is compact whenever $2 \leq N<p<+\infty$.

Here we consider the following functionals:

- $F_{i}(\xi):=\int_{0}^{\xi} f_{i}(t) d t$ for every $\xi \in \mathbb{R}$.

- $\Phi(u, v):=\frac{\|u\|_{r}^{p}}{p}+\frac{\|v\|_{r}^{q}}{q}$ for every $(u, v) \in X$.

- $\Psi(u, v):=\int_{\mathbb{R}^{\mathbb{N}}} \alpha_{1} F_{1}(v(x)) d x+\int_{\mathbb{R}^{\mathbb{N}}} \alpha_{2} F_{2}(u(x)) d x$, for every $(u, v) \in X$. 
- $I_{\lambda}(u, v):=\Phi(u, v)-\lambda \Psi(u, v)$ for every $(u, v) \in X$.

By standard arguments [5], we can show that $\Phi$ is Gâteaux differentiable, coercive and sequentially weakly lower semicontinuous whose derivative at the point $(u, v) \in X$ is the functional $\Phi^{\prime}(u, v) \in X^{*}$ given by

$$
\Phi^{\prime}(u, v)(z, w)=\left(\int_{\mathbb{R}^{\mathbb{N}}}|\nabla u|^{p-2} \nabla u . \nabla z d x+\int_{\mathbb{R}^{\mathbb{N}}}|u|^{p-2} u z d x\right)+\left(\int_{\mathbb{R}^{\mathbb{N}}}|\nabla v|^{q-2} \nabla v \cdot \nabla w d x+\int_{\mathbb{R}^{\mathbb{N}}}|v|^{q-2} v w d x\right),
$$

for every $(z, w) \in X$. Also standard arguments show that the functional $\Psi_{i}$ are well defined, sequentially weakly upper semicontinuous and Gâteaux differentiable whose Gâteaux derivative at the point $(u, v) \in X$ and for every $(z, w) \in X$ is given by,

$$
\Psi^{\prime}(u, v)(z, w)=\int_{\mathbb{R}^{\mathbb{N}}} \alpha_{1}(x) f_{1}(u(x)) d x+\int_{\mathbb{R}^{\mathbb{N}}} \alpha_{2}(x) f_{2}(v(x)) d x .
$$

\section{Weak solutions}

First we recall the following theorem [3, Theorem 2.1].

Theorem 2.1. Let $X$ be a reflexive real Banach space, $\Phi, \Psi: X \rightarrow \mathbb{R}$ be two Gâteaux differentiable functionals such that $\Phi$ is sequentially weakly lower semicontinuous, strongly continuous and coercive, and $\Psi$ is sequentially weakly upper semicontinuous. For every $r>\inf _{X} \Phi$, set

$$
\varphi(r):=\inf _{\Phi(u)<r} \frac{\sup _{\Phi(v)<r} \Psi(v)-\Psi(u)}{r-\Phi(u)}, \gamma:=\liminf _{r \rightarrow+\infty} \varphi(r), \quad \text { and } \quad \delta:=\liminf _{r \rightarrow\left(\inf _{X} \Phi\right)^{+}} \varphi(r) .
$$

Then the following properties hold:

(a) for every $r>\inf _{X} \Phi$ and every $\left.\lambda \in\right] 0, \frac{1}{\varphi(r)}$, the restriction of the functional $I_{\lambda}:=\Phi-\lambda \Psi$ to $\Phi^{-1}(]-\infty, r[)$ admits a global minimum, which is a critical point (local minimum) of $I_{\lambda}$ in $X$.

(b) if $\gamma<+\infty$, then for each $\lambda \in] 0, \frac{1}{\gamma}$, the following alternative holds either,

(b) $I_{\lambda}$ possesses a global minimum, or

(b) there is a sequence $\left\{u_{n}\right\}$ of critical points (local minima) of $I_{\lambda}$ such that $\lim _{n \rightarrow+\infty} \Phi\left(u_{n}\right)=+\infty$.

(c) if $\delta<+\infty$, then for each $\lambda \in] 0, \frac{1}{\delta}$, the following alternative holds either:

$\left(c_{1}\right)$ there is a global minimum of $\Phi$ which is a local minimum of $I_{\lambda}$. or,

$\left(c_{2}\right)$ there is a sequence $\left\{u_{n}\right\}$ of pairwise distinct critical points (local minima) of $I_{\lambda}$ which weakly converges to a global minimum of $\Phi$, with $\lim _{n \rightarrow+\infty} \Phi\left(u_{n}\right)=\inf _{u \in X} \Phi(u)$.

For fixed $D>0$, set

$$
m(D):=\operatorname{meas}(B(0, D))=D^{N} \frac{\pi^{\frac{N}{2}}}{\Gamma\left(1+\frac{N}{2}\right)},
$$

where $\Gamma$ is the Gamma function defined by $\Gamma(t):=\int_{0}^{+\infty} z^{t-1} e^{-z} d z$ for all $t>0$. Moreover,

$$
\Omega:=\max \left\{\frac{m(D)\left(\frac{\sigma(N, p)}{D^{p}}+g(p, N)\right)}{p \lambda B_{1}\left\|\alpha_{2}\right\|_{B\left(0, \frac{D}{2}\right)}}, \frac{m(D)\left(\frac{\sigma(N, q)}{D^{q}}+g(q, N)\right)}{q \lambda B_{2}\left\|\alpha_{1}\right\|_{B\left(0, \frac{D}{2}\right)}}\right\}>0,
$$

where $\sigma(N, p):=2^{p-N}\left(2^{N}-1\right), c=\frac{2 p}{2-N}, m_{1}, m_{0}$ are upper and lower bounds for $M(t)$ in (1) and

$$
g(p, N):=\frac{1+2^{N+p} N \int_{\frac{1}{2}}^{1} t^{N-1}(1-t)^{p} d t}{2^{N}} .
$$

Assume $\|\cdot\|_{1}$ denotes the norm of $L^{1}(\Omega)$ and $F(\xi):=F_{1}(\xi)+F_{2}(\xi)$.

Theorem 2.2. Let $f_{i}: \mathbb{R} \rightarrow \mathbb{R}$ be two continuous and radially symmetric functions. Set

$$
A:=\liminf _{\left(\xi_{1}, \xi_{2}\right) \rightarrow+\infty} \frac{\max _{\left|t_{1}\right| \leq \xi_{1}} F_{2}\left(t_{1}\right)}{\left|\xi_{1}\right|^{p}}+\frac{\max _{\left|t_{2}\right| \leq \xi_{2}} F_{1}\left(t_{2}\right)}{\left|\xi_{2}\right|^{q}}, \quad B_{1}:=\limsup _{\xi_{2} \rightarrow+\infty} \frac{F_{1}\left(\xi_{2}\right)}{\left|\xi_{2}\right|^{p}} \text {, and } B_{2}:=\limsup _{\xi_{1} \rightarrow+\infty} \frac{F_{2}\left(\xi_{1}\right)}{\left|\xi_{1}\right|^{q}} \text {. }
$$

where $B:=B_{1}+B_{2}, \xi=\left(\xi_{1}, \xi_{2}\right)$. If $\inf _{\left(\xi_{1}, \xi_{2}\right) \geq 0} F_{2}\left(\xi_{1}\right)+F_{1}\left(\xi_{2}\right)=0$ and $A<\Omega m_{0} B$, where $\Omega$ is given by (3), for every

$$
\lambda \in \Lambda:=] \Omega, \frac{1}{\left(p c_{1}^{p}\left\|\alpha_{2}\right\|_{1}+q c_{2}^{q}\left\|\alpha_{1}\right\|_{1}\right) A}[,
$$

there exists an unbounded sequence of radially symmetric weak solutions for (1) in X. 
Proof. For fixed $\lambda \in \Lambda$, we consider $\Phi, \Psi$ and $I_{\lambda}$ as in the last section. Knowing that $\Phi$ and $\Psi$ satisfy the regularity assumptions in Theorem 2.1. In order to study the critical points of $I_{\lambda}$ in $X$, we show that $\lambda<\frac{1}{\gamma}<+\infty$, where $\gamma=$ $\liminf _{r \rightarrow+\infty} \phi(r)$. Let $\left\{t_{n}\right\}$ be a sequence of positive numbers such that $\lim _{n \rightarrow \infty} t_{n}=+\infty, r_{1 n}:=\frac{t_{1 n}^{p}}{p c_{1}^{p}}$ and $r_{2 n}:=\frac{t_{2 n}^{q}}{q c_{2}^{q}}$, for all $n \in \mathbb{N}$. Set $r_{n}=\min \left\{r_{n 1}, r_{n 2}\right\}$. Considering Theorem 1.2 (by relation (2)), a computation shows that

$$
\begin{aligned}
\Phi^{-1}(]-\infty, r_{n}[) & =\left\{(z, w) \in X: \Phi(z, w)<r_{n}\right\} \\
& =\left\{(z, w) \in X: \frac{\|z\|_{r}^{p}}{p}+\frac{\|w\|_{r}^{q}}{q}<r_{n}\right\} \\
& \subset\left\{(z, w) \in X ;\|(z, w)\|_{\infty}<t_{n}\right\},
\end{aligned}
$$

where $t_{n}=\min \left\{t_{n 1}, t_{n 2}\right\}$.

Since $\Phi(0,0)=\Psi(0,0)=0$, by a computation one can show

$$
\varphi\left(r_{n}\right)=\inf _{\Phi(u, v)<r_{n}} \frac{\left(\sup _{\Phi(z, w)<r_{n}} \Psi(z, w)\right)-\Psi(u, v)}{r_{n}-\Phi(u, v)} \leq\left(p c_{1}^{p}\left\|\alpha_{2}\right\|_{1}+q c_{2}^{q}\left\|\alpha_{1}\right\|_{1}\right) A .
$$

Hence

$$
\gamma \leq \liminf _{n \rightarrow+\infty} \varphi\left(r_{n}\right) \leq\left(p c_{1}^{p}\left\|\alpha_{2}\right\|_{1}+q c_{2}^{q}\left\|\alpha_{1}\right\|_{1}\right) A<+\infty .
$$

Now, we show that $I_{\lambda}$ is unbounded from below. Let $\left\{d_{1 n}\right\}$ and $\left\{d_{2 n}\right\}$ be two sequences of positive numbers such that $\lim _{n \rightarrow+\infty} d_{1 n}=\lim _{n \rightarrow+\infty} d_{2 n}=+\infty$ and

$$
B_{1}=\lim _{n \rightarrow+\infty} \frac{F_{1}\left(d_{2 n}\right)}{d_{2 n}^{q}}, B_{2}=\lim _{n \rightarrow+\infty} \frac{F_{2}\left(d_{1 n}\right)}{d_{1 n}^{p}}
$$

Define $\left\{\left(H_{1 n}, H_{2 n}\right)\right\} \in X$ by

$$
H_{\text {in }}(x):= \begin{cases}0 & \mathbb{R}^{\mathbb{N}} \backslash B(0, D) \\ d_{\text {in }} & B\left(0, \frac{D}{2}\right) \\ \frac{2 d_{\text {in }}}{D}(D-|x|) & B(0, D) \backslash B\left(0, \frac{D}{2}\right),\end{cases}
$$

for every $n \in \mathbb{N}$ and $i=1,2$. By a similar argument and computations in [5, P.1017] one can show that

$$
\left\|H_{2 n}\right\|_{r}^{q}=d_{2 n}^{q} m(D)\left(\frac{\sigma(N, p)}{D^{q}}+g(q, N)\right) \text { and }\left\|H_{1 n}\right\|_{r}^{p}=d_{1 n}^{p} m(D)\left(\frac{\sigma(N, p)}{D^{p}}+g(p, N)\right) .
$$

Condition $(i)$, implies

$$
\begin{gathered}
\int_{\mathbb{R}^{\mathbb{N}}} \alpha_{1}(x) F_{1}\left(H_{2 n}(x)\right) d x \geq \int_{B\left(0, \frac{D}{2}\right)} \alpha_{1}(x) F_{1}\left(d_{2 n}\right) d x=F_{1}\left(d_{2 n}\right)\left\|\alpha_{1}\right\|_{B\left(0, \frac{D}{2}\right)}, \text { and } \\
\int_{\mathbb{R}^{\mathbb{N}}} \alpha_{2}(x) F_{2}\left(H_{1 n}(x)\right) d x \geq \int_{B\left(0, \frac{D}{2}\right)} \alpha_{2}(x) F_{2}\left(d_{1 n}\right) d x=F_{2}\left(d_{1 n}\right)\left\|\alpha_{2}\right\|_{B\left(0, \frac{D}{2}\right)},
\end{gathered}
$$

for every $n \in N$. Then

$$
\begin{aligned}
I_{\lambda}\left(H_{1 n}, H_{2 n}\right)= & \Phi\left(H_{1 n}, H_{2 n}\right)-\lambda \Psi\left(H_{1 n}, H_{2 n}\right) \\
= & \frac{\left\|H_{1 n}\right\|_{r}^{p}}{p}+\frac{\left\|H_{2 n}\right\|_{r}^{q}}{q}-\lambda \int_{\mathbb{R}^{N}} \alpha_{1}(x) F_{1}\left(H_{2 n}(x)\right) d x-\lambda \int_{\mathbb{R}^{N}} \alpha_{2}(x) F_{2}\left(H_{1 n}(x)\right) d x \\
\leq & \frac{d_{1 n}^{p} m(D)\left(\frac{\sigma(N, p)}{D^{P}}+g(p, N)\right)}{p}+\frac{d_{2 n}^{q} m(D)\left(\frac{\sigma(N, q)}{D^{q}}+g(q, N)\right)}{q} \\
& -\lambda\left(F_{1}\left(d_{2 n}\right)\left\|\alpha_{1}\right\|_{B\left(0, \frac{D}{2}\right)}+F_{2}\left(d_{1 n}\right)\left\|\alpha_{2}\right\|_{B\left(0, \frac{D}{2}\right)} \cdot\right)
\end{aligned}
$$

If $B<+\infty\left(B_{1}, B_{2}<+\infty\right)$, the conditions (5) implies that

there exists $N_{1}$ such that for all $n \geq N_{1}$ we have $F_{1}\left(d_{2 n}\right)>\varepsilon B_{1} d_{2 n}^{p}$, and there exists $N_{2}$ such that for all $n \geq N_{2}$ we have $F_{2}\left(d_{1 n}\right)>\varepsilon B_{2} d_{1 n}^{q}$.

Then for every $n \geq N_{\varepsilon}:=\max \left\{N_{1}, N_{2}\right\}$,

$$
I_{\lambda}\left(H_{1 n}, H_{2 n}\right) \leq \frac{d_{1 n}^{p} m(D)\left(\frac{\sigma(N, p)}{D^{P}}+g(p, N)\right)}{p}+\frac{d_{2 n}^{q} m(D)\left(\frac{\sigma(N, q)}{D^{q}}+g(q, N)\right)}{q}-\lambda \varepsilon\left(d_{1 n}^{p} B_{2}\left\|\alpha_{1}\right\|_{B\left(0, \frac{D}{2}\right)}+d_{2 n}^{q} B_{2}\left\|\alpha_{1}\right\|_{B\left(0, \frac{D}{2}\right)}\right)
$$




$$
=d_{1 n}^{p}\left(\frac{m(D)\left(\frac{\sigma(N, p)}{D^{P}}+g(p, N)\right)}{p}-\lambda \varepsilon B_{1}\left\|\alpha_{2}\right\|_{B\left(0, \frac{D}{2}\right)}\right)+d_{2 n}^{q}\left(\frac{m(D)\left(\frac{\sigma(N, q)}{D^{q}}+g(q, N)\right)}{q}-\lambda \varepsilon B_{2}\left\|\alpha_{1}\right\|_{B\left(0, \frac{D}{2}\right)}\right) .
$$

If we set

$$
\Omega:=\max \left\{\frac{m(D)\left(\frac{\sigma(N, p)}{D^{p}}+g(p, N)\right)}{p \lambda B_{1}\left\|\alpha_{2}\right\|_{B\left(0, \frac{D}{2}\right)}}, \frac{m(D)\left(\frac{\sigma(N, q)}{D^{q}}+g(q, N)\right)}{q \lambda B_{2}\left\|\alpha_{1}\right\|_{B\left(0, \frac{D}{2}\right)}}\right\},
$$

then for $\varepsilon \in(\Omega, 1)$ one can get $\lim _{n \rightarrow+\infty} I_{\lambda}\left(H_{1 n}, H_{2 n}\right)=-\infty$.

If at least one of the $B_{1}$ or $B_{2}$ are $+\infty$. Let $B_{1}=+\infty$, and consider $M_{1}>\Omega$, then by (5) there exists $N_{M_{1}}$ such that for every $n>N_{M_{1}}$, we have $F_{1}\left(d_{1 n}\right)>M_{1} d_{1 n}^{p}$. Moreover, for every $n>N_{M_{1}}$

$$
\begin{aligned}
I_{\lambda}\left(H_{1 n}, H_{2 n}\right) & \leq \frac{d_{1 n}^{p} m(D)\left(\frac{\sigma(N, p)}{D^{p}}+g(p, N)\right)}{p}+\frac{d_{2 n}^{q} m(D)\left(\frac{\sigma(N, q)}{D^{q}}+g(q, N)\right)}{q}-\lambda\left(d_{1 n}^{p} M_{1}\left\|\alpha_{2}\right\|_{B\left(0, \frac{D}{2}\right)}+d_{2 n}^{q} M_{1}\left\|\alpha_{1}\right\|_{B\left(0, \frac{D}{2}\right)}\right) \\
& =d_{1 n}^{p}\left(\frac{m(D)\left(\frac{\sigma(N, p)}{D^{P}}+g(p, N)\right)}{p}-\lambda M_{1}\left\|\alpha_{2}\right\|_{B\left(0, \frac{D}{2}\right)}\right)+d_{2 n}^{q}\left(\frac{m(D)\left(\frac{\sigma(N, q)}{D^{q}}+g(q, N)\right)}{q}-\lambda M_{1}\left\|\alpha_{1}\right\|_{B\left(0, \frac{D}{2}\right)}\right) .
\end{aligned}
$$

This implies that $\lim _{n \rightarrow+\infty} I_{\lambda}\left(H_{1 n}, H_{2 n}\right)=-\infty$.

Now, Theorem 2.1(b) implies, the functional $I_{\lambda}$ admits an unbounded sequence $\left\{u_{n}\right\} \subset X$ of critical points. Considering Theorem 1.1, these critical points are also critical points for the smooth and $O(N)$-invariant functional $I_{\lambda}: W^{1, p}\left(\mathbb{R}^{N}\right) \rightarrow$ $\mathbb{R}$. Therefore, there is a sequence of radially symmetric weak solutions for the problem (1), which are unbounded in $W^{1, p}\left(\mathbb{R}^{N}\right)$.

Here we prove our second result which says that under different conditions the problem (1) has a sequence of weak solutions, which converges weakly to zero.

Theorem 2.3. Let $f_{i}: \mathbb{R} \rightarrow \mathbb{R}$ be two continuous and radially symmetric functions. Set

$$
A^{\prime}:=\liminf _{\left(\xi_{1}, \xi_{2}\right) \rightarrow 0^{+}} \frac{\max _{\left|t_{1}\right| \leq \xi_{1}} F_{2}\left(t_{1}\right)}{\left|\xi_{1}\right|^{p}}+\frac{\max _{\left|t_{2}\right| \leq \xi_{2}} F_{1}\left(t_{2}\right)}{\left|\xi_{2}\right|^{q}}, \quad B_{1}^{\prime}:=\limsup _{\xi_{2} \rightarrow 0^{+}} \frac{F_{1}\left(\xi_{2}\right)}{\left|\xi_{2}\right|^{p}}, \text { and } B_{2}^{\prime}:=\limsup _{\xi_{1} \rightarrow 0^{+}} \frac{F_{2}\left(\xi_{1}\right)}{\left|\xi_{1}\right|^{q}},
$$

where $B^{\prime}:=B_{1}^{\prime}+B_{2}^{\prime}, \xi=\left(\xi_{1}, \xi_{2}\right)$. If $\inf _{\left(\xi_{1}, \xi_{2}\right) \geq 0} F_{2}\left(\xi_{1}\right)+F_{1}\left(\xi_{2}\right)=0$ and $A^{\prime}<\Omega m_{0} B^{\prime}$, where $\Omega$ is given by (3), for every

$$
\left.\lambda \in \Lambda^{\prime}:=\right] \Omega, \frac{1}{\left(p c_{1}^{p}\left\|\alpha_{2}\right\|_{1}+q c_{2}^{q}\left\|\alpha_{1}\right\|_{1}\right) A^{\prime}}[,
$$

there exists an unbounded sequence of radially symmetric weak solutions for (1) in X.

Proof. For fixed $\lambda \in \Lambda^{\prime}$, we consider $\Phi, \Psi$ and $I_{\lambda}$ as in Section 2. Knowing that $\Phi$ and $\Psi$ satisfy the regularity assumptions in Theorem 2.1, we show that $\lambda<\frac{1}{\delta}$. We know that $\inf _{X} \Phi=0$. Set $\delta:=\liminf _{r \rightarrow 0^{+}} \varphi(r)$. A computation similar to the one in the Theorem 2.2 implies $\delta<\infty$ and if $\lambda \in \Lambda^{\prime}$ then $\lambda<\frac{1}{\delta}$. A compaction (similar in the Theorem 2.2) shows that $I_{\lambda}\left(H_{1 n}, H_{2 n}\right)<0$ for $n$ large enough and thus zero is not a local minimum of $I_{\lambda}$. Therefore, there exists a sequence $\left\{u_{n}\right\} \subset X$ of critical points of $I_{\lambda}$ which converges weakly to zero in $X$ as $\lim _{n \rightarrow+\infty} \Phi\left(u_{n}\right)=0$.

Again, considering Theorem 1.1, these critical points are also critical points for the smooth and $O(N)$-invariant functional $I_{\lambda}: W^{1, p}\left(\mathbb{R}^{N}\right) \rightarrow \mathbb{R}$. Therefore, there is a sequence of radially symmetric weak solutions for the problem (1), which converges weakly to zero in $W^{1, p}\left(\mathbb{R}^{N}\right)$.

\section{Acknowledgment}

This work is supported by I.N.D.A.M - G.N.A.M.P.A. 2019 and the "RUDN University Program 5-100".

\section{References}

[1] F. Behboudi, A. Razani, Two weak solutions for a singular ( $p, q)$-Laplacian problem, Filomat 33 (2019) 3399-3407.

[2] S. Benbernou, S. Gala, M. A. Ragusa, On the regularity criteria for the $3 D$ magnetohydrodynamic equations via two components in terms of BMO space, Math. Methods Appl. Sci. 37 (2014) 2320-2325.

[3] G. Bonanno, G. Molica Bisci, Infinitely many solutions for a boundary value problem with discontinuous nonlinearities, Bound. Value Probl. 2009 (2009) Art\# 670675.

[4] H. Brezis, Analyse fonctionnelle: Théorie et applications, Masson, Paris, 1963.

[5] P. Candito, G. Molica Bisci, Radially symmetric weak solutions for elliptic problems in $\mathbb{R}^{N}$, Differential Integral Equations 26 (2013) $1009-1026$. 
[6] Q. Chen, C. Chen, Infinitely many solutions for a class of $p$-biharmonic equation in $\mathbb{R}^{N}$, Bull. Iranian Math. Soc. 43 (2017) $205-215$.

[7] D. Ehsani, M. Mokhtarzadeh, A. Razani, Expansions of Dirichlet to Neumann operators under perturbations, Appl. Anal. 89 (2010) 789-799.

[8] S. Gala, Q. Liu, M. A. Ragusa, A new regularity criterion for the nematic liquid crystal flows, Appl. Anal. 91 (2012) 1741-1747.

[9] S. Gala, M. A. Ragusa, A logarithmically improved regularity criterion for the 3D MHD equations in Morrey-Campanato space, AIMS Math. 2 (2017) 16-23.

[10] S. Gala, M. A. Ragusa, Logarithmically improved regularity criterion for the Boussinesq equations in Besov spaces with negative indices, Appl. Anal. 95 (2016) 1271-1279.

[11] M. Hesaaraki, A. Razani, Detonative travelling waves for combustion, Appl. Anal. 77 (2001) 405-418.

[12] M. Hesaaraki, A. Razani, On the existence of Chapman-Jouguet detonation Waves, Bull. Aust. Math. Soc. 63 (2001) $485-496$.

[13] S. M. Khalkhali, S. Heidarkhani, A. Razani, Infinitely many solutions for a fourth-order boundary-value problem, Electron. J. Differential Equations 2012 (2012) Art\# 164.

[14] S. M. Khalkhali, A. Razani, Multiple solutions for a quasilinear ( $p, q)$-elliptic system, Electron. J. Differential Equations 2013 (2013) Art\# 144.

[15] A. Kristály, Infinitely many solutions for a differential inclusion problem in $\mathbb{R}^{N}$, J. Differential Equations 220 (2006) 511-530.

[16] A. Kristály, V. D. Rădulescu, C. G. Varga, Variational Principles in Mathematical Physics, Geometry, and Conomics: Qualitative Analysis of Nonlinear Equations and Unilateral Problems (Encyclopedia of Mathematics and its Applications, Vol. 136), Cambridge University Press, Cambridge, 2010.

[17] Y. Li, Multiple solutions for perturbed $p$-Lalacian problems on $\mathbb{R}^{N}$, Electron. J. Differential Equations 2015 (2015) Art\# 23.

[18] E. Lindgren, A. Razani, The $N$-membranes problem, Bull. Iranian Math. Soc. 35 (2009) 31-40.

[19] R. Mahdavi Khanghahi, A. Razani, Solutions for a singular elliptic problem involving the $p(x)$-Laplacian, Filomat 32 (2018) $4841-4850$.

[20] M. Makvand Chaharlang, M. A. Ragusa, A. Razani, A sequence of radially symmetric weak solutions for some nonlocal elliptic problem in $\mathbb{R}^{N}$, Mediterr. J. Math. 17 (2020) Art\# 53.

[21] M. Makvand Chaharlang, A. Razani, A fourth order singular elliptic problem involving $p$-biharmonic operator, Taiwanese J. Math. 23 (2019) 589599.

[22] M. Makvand Chaharlang, A. Razani, Existence of infinitely many solutions for a class of nonlocal problems with Dirichlet boundary condition, Commun. Korean Math. Soc 34 (2019) 155-167.

[23] M. Makvand Chaharlang, A. Razani, Infnitely many solutions for a fourth order singular elliptic problem, Filomat 32 (2018) 5003-5010.

[24] M. Makvand Chaharlang, A. Razani, Two weak solutions for some Kirchhoff-type problem with Neumann boundary condition, Georgian Math. J., DOI: 10.1515/gmj-2019-2077, In press.

[25] M. R. Mokhtarzadeh, M. R. Pournaki, A. Razani, A note on periodic solutions of Riccati equations, Nonlinear Dyn. 62 (2010) 119-125.

[26] M. R. Mokhtarzadeh, M. R. Pournaki, A. Razani, An existence-uniqueness theorem for a class of boundary value problems, Fixed Point Theory 13 (2012) 583-592.

[27] R. S. Palais, The principle of symmetric criticality, Commun. Math. Phys. 69 (1979) 19-30.

[28] S. Polidoro, M. A. Ragusa, Harnack inequality for hypoelliptic ultraparabolic equations with a singular lower order term, Rev. Mat. Iberoam 24 (2008) 1011-1046.

[29] M. R. Pournaki, A. Razani, Erratum to "On the existence of periodic solutions for a class of generalized forced Liénard equations" [Appl. Math. Lett. 20(3) (2007) 248-254], Appl. Math. Lett. 21 (2008) 880.

[30] M. R. Pournaki, A. Razani, On the existence of periodic solutions for a class of generalized forced Liénard equations, Appl. Math. Lett. 20 (2007) $248-254$.

[31] M. A. Ragusa, Local Hölder regularity for solutions of elliptic systems, Duke Math. J. 113 (2002) 385-397.

[32] M. A. Ragusa, On weak solutions of ultraparabolic equations, Nonlinear Anal. 47 (2001) 503-511.

[33] M. A. Ragusa, Parabolic Herz spaces and their applications, Appl. Math. Lett. 25 (2012) 1270-1273.

[34] M. A. Ragusa, A Tachikawa, Regularity for minimizers for functionals of double phase with variable exponents, Adv. Nonlinear Anal. 9 (2020) $710-728$.

[35] A. Razani, An existence theorem for ordinary differential equation in Menger probabilistic metric space, Miskolc Math. Notes 15 (2014) $711-716$.

[36] A. Razani, Chapman-Jouguet detonation profile for a qualitative model, Bull. Aust. Math. Soc. 66 (2002) 393-403.

[37] A. Razani, Chapman-Jouguet travelling wave for a two-steps reaction scheme, Ital. J. Pure Appl. Math. 39 (2018) 544-553.

[38] A. Razani, Existence of Chapman-Jouguet detonation for a viscous combustion model, J. Math. Anal. Appl. 293 (2004) 551-563.

[39] A. Razani, On the existence of premixed laminar flames, Bull. Aust. Math. Soc. 69 (2004) 415-427.

[40] A. Razani, Shock waves in gas dynamics, Surv. Math. Appl. 2 (2007) 59-89.

[41] A. Razani, Structure of shock wave for a viscous combustion model, Filomat 26 (2012) 389-395.

[42] A. Razani, Subsonic detonation waves in porous media, Phys. Scr. 94 (2019) Art\# 085209.

[43] A. Razani, Weak and strong detonation profiles for a qualitative model, J. Math. Anal. Appl. 276 (2002) 868-881.

[44] A. Razani, Z. Goodarzi, A solution of Volterra-Hamerstain integral equation in partially ordered sets, Int. J. Ind. Math. 3 (2011) 277-281.

[45] A. Scapellato, Regularity of solutions to elliptic equations on Herz spaces with variable exponents, Bound. Value Probl. 2019 (2029) Art\# 2.

[46] W. A. Strauss, Existence of solitary waves in higher dimensions, Comm. Math. Phys. 55 (1977) 149-162.

[47] M. Willem, Minimiax Theorems (Progress in Nonlinear Differential Equations and Their Applications, Vol. 24 ), Birkhäuser, Boston, 1996.

[48] R. Yang, W. Zhang, X. Liu, Sign-changing solutions for $p$-biharmonic equations with Hardy potential in $\mathbb{R}^{N}$, Acta Math. Sci. Ser. B 37 (2017) 593-606. 